\title{
Phonon Lifetimes throughout the Brillouin Zone at Elevated Temperatures from Experiment and $A b$ Initio
}

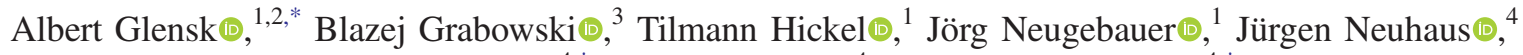 \\ Klaudia Hradil $\odot,{ }^{4, \dagger}$ Winfried Petry $\odot{ }^{4}{ }^{4}$ and Michael Leitner $\oplus^{4, \ddagger}$ \\ ${ }^{1}$ Max-Planck-Institut für Eisenforschung GmbH, 40237 Düsseldorf, Germany \\ ${ }^{2}$ Laboratory for Multiscale Mechanics and Modeling (LAMMM) and Laboratory for Computational Science and Modelling (COSMO), \\ Ecole Polytechnique Federale de Lausanne, 1015 Lausanne, Switzerland \\ ${ }^{3}$ Institute of Materials Science, University of Stuttgart, Pfaffenwaldring 55, 70569 Stuttgart, Germany \\ ${ }^{4}$ Heinz Maier-Leibnitz Zentrum (MLZ), Technische Universität München, Lichtenbergstr. 1, 85748 Garching, Germany
}

(Received 15 July 2019; published 2 December 2019)

\begin{abstract}
We obtain phonon lifetimes in aluminium by inelastic neutron scattering experiments, by ab initio molecular dynamics, and by perturbation theory. At elevated temperatures significant discrepancies are found between experiment and perturbation theory, which disappear when using molecular dynamics due to the inclusion of full anharmonicity and the correct treatment of the multiphonon background. We show that multiple-site interactions are small and that local pairwise anharmonicity dominates phonon-phonon interactions, which permits an efficient computation of phonon lifetimes.
\end{abstract}

DOI: $10.1103 /$ PhysRevLett.123.235501

Thermodynamic material properties are nowadays routinely accessible from $a b$ initio to $\mathrm{meV}$ precision up to the melting temperature [1-3]. In contrast to such integral quantities - which are ensemble averages over the phase space as, e.g., the Gibbs free energy-phonon properties are spectrally resolved and, therefore, pose a greater challenge for $a b$ initio simulations. Phonon frequencies and lifetimes are the key input to thermal conductivity and among the most sensitive measures of anharmonicity in solids. Phonon lifetimes have been successfully calculated at room temperature from the third-order force constant tensors employing perturbation theory (PT) [4-9] and recently extended to higher orders [10]. At elevated temperatures, however, one typically has to accept differences on the order of a factor of two between state-of-the-art first principles predictions and measured data $[6,11,12]$.

Experimental determinations of phonon linewidths $\Gamma$ (which are inversely related to phonon lifetimes as $\tau=1 / \pi \Gamma)$ employ for instance Raman and infrared spectroscopy, which are highly accurate but restricted to the $\Gamma$ point [13]. Probing the linewidth over the full Brillouin zone requires to use neutrons or $\mathrm{x}$ rays (specifically for small sample volumes [14]) as probes, where the focus lies often on electron-phonon scattering $[12,15]$ or defect scattering [16]. In contrast, investigations of anharmonic

Published by the American Physical Society under the terms of the Creative Commons Attribution 4.0 International license. Further distribution of this work must maintain attribution to the author(s) and the published article's title, journal citation, and DOI. phonon-phonon scattering over large regions of reciprocal space for unary elements at high temperatures have been reported up to now only for $\mathrm{K}$ [17], $\mathrm{Li}$ [18], and $\mathrm{Cu}$ [19].

In this Letter we report fully q-dependent phonon linewidths in $\mathrm{Al}$ by means of inelastic neutron scattering up to the melting point. Within the framework of densityfunctional theory (DFT) we perform state-of-the-art firstorder perturbation theory as well as molecular dynamics (MD) and compare the resulting linewidths. At the hereinvestigated elevated temperatures, quantum effects are small, so that classical MD is expected to capture the essential physics. In contrast to perturbative approaches, MD has the advantage to fully include all orders of phononphonon scattering, and, further, that the availability of the actual spectral function permits a direct comparison to experiment. We show that a quantitative agreement between experiment and theory at elevated temperatures can only be achieved from molecular dynamics since it probes (i) the full anharmonicity and (ii) naturally occurring contributions due to multiphonon background scattering. We unveil the dominant mechanism controlling phonon lifetimes in $\mathrm{Al}$ and utilize this knowledge for the computation of lifetimes including the full anharmonicity at significantly reduced computational cost.

The inelastic neutron scattering measurements have been performed at the thermal triple-axis spectrometer PUMA at the Heinz Maier-Leibnitz Zentrum (MLZ), Garching [20,21]. For our DFT calculations we employ VASP [22,23], projector augmented-wave potentials [24], the generalized gradient approximation (GGA), as well as the local density approximation (LDA) [25-27] at the corresponding self-consistent lattice constants. Further, we 

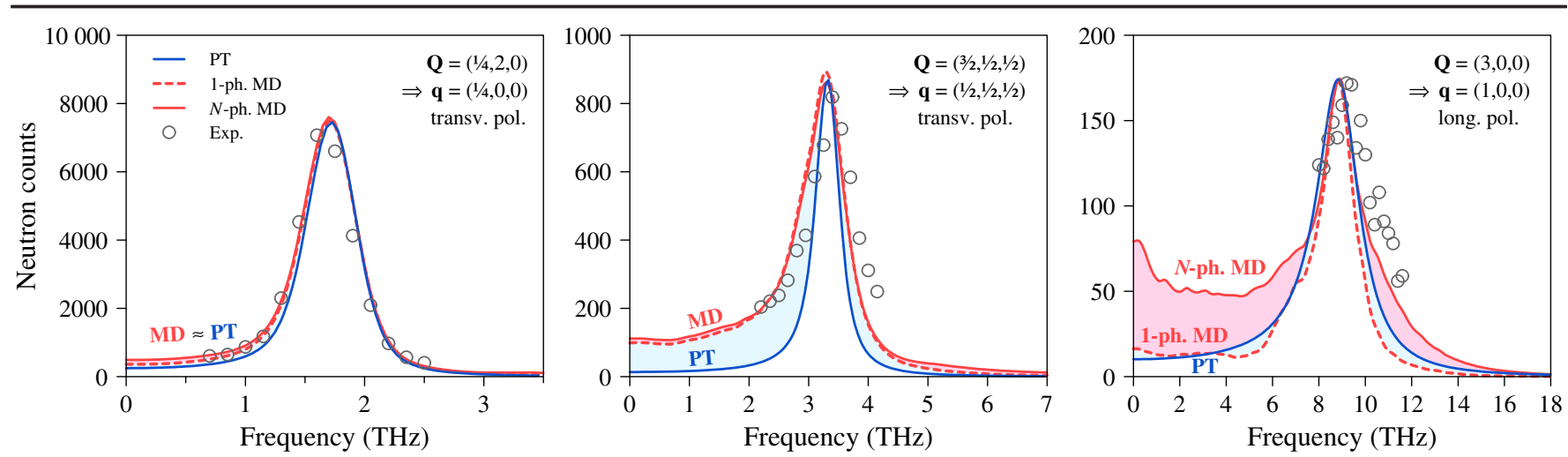

FIG. 1. Inelastic neutron spectra (circles) along with theoretical spectral densities according to perturbation theory (blue lines), onephonon MD [dashed red lines-Eq. (1)] and $N$-phonon MD spectral densities [solid red lines-Eq. (3)] of Al at $900 \mathrm{~K}$. The chosen spectra are representative for the cases where either PT is adequate (left panel: $\mathbf{q}=(0.25,0,0)$ with dominant transversal contribution), where it severely underestimates anharmonic broadening (middle panel: $L$ point with dominant transversal contribution), or where the $N$-phonon background appreciably affects the resulting spectrum (right panel: $\mathrm{X}$ point with longitudinal polarization). Note that the theoretical spectra have been convolved with the experimental resolution as detailed in the Supplemental Material [29], corresponding to Gaussian kernels with FWHMs of $0.37,0.22$, and $0.34 \mathrm{THz}$ in the left, middle, and right panels, respectively.

perform perturbation theory calculations as implemented in PHONO3PY $[9,28]$. Convergence errors due to DFT related parameters (e.g., supercell size, $\mathbf{k}$ points, energy cutoff, time step, etc.) have been carefully checked and are summarized in the Supplemental Material [29].

Our first main result is shown in Fig. 1, where we compare our raw experimental spectra at $900 \mathrm{~K}$ for selected scattering vectors to DFT perturbation theory: for some cases, the experimental spectra (empty circles) are significantly broader than the spectra predicted by perturbation theory (blue lines). We note that also for copper-which is more anharmonic than $\mathrm{Al}$ [3]—experimental linewidths have been reported to be twice as large as calculations using first-principles perturbation theory [6]. To analyze the discrepancies found in $\mathrm{Al}$, it is desirable to go beyond the perturbative treatment to account for the full set of anharmonic interactions which become important at elevated temperatures.

The systematic inclusion of higher orders in perturbation theory is, however, a significant numerical challenge for first principles and only very recently the effects of fourphonon scattering on phonon lifetimes have been investigated [10]. In contrast, molecular dynamics calculations capture the full set of phonon-phonon interactions, but have the drawback of increased computational demands. Consequently, previous studies on phonon lifetimes employing MD had to resort to empirical potentials $[32,33]$ that are typically not optimized for reproducing the anharmonic characters of atomic interactions, which is the key quantity controlling phonon broadening.

In this study, we perform extensive ab initio DFT MD for bulk aluminium and extract for every relevant $\mathbf{q}$ vector in the Brillouin zone the phonon frequency $\nu_{0}$ and linewidth $\Gamma$ (full width at half maximum-FWHM) from the corresponding power spectral density (PSD)—also known as scattering function or power spectrum (exemplary spectra are illustrated in Fig. 1). Conventionally, this is done by first computing the scattering amplitude $A(\mathbf{q}, t)$ as the projection of the atomic displacements onto the normal mode coordinates of the respective phonons

$$
A(\mathbf{q}, t) \propto \sum_{j}\left[\mathbf{r}_{j}(t)-\mathbf{r}_{j}^{\mathrm{eq}}\right] \mathbf{p} e^{-i \mathbf{q} \mathbf{r}_{j}^{\mathrm{eq}}}
$$

where $\mathbf{r}_{j}(t)$ is the position of atom $j$ at time $t$ during the simulation run, $\mathbf{r}_{j}^{\text {eq }}$ is its ideal position on the lattice and $\mathbf{p}$ is the phonon polarization vector [33]. Fourier-transforming the absolute-squared amplitude [Eq. (1)] over time then gives the power spectrum.

For harmonic interactions, a single phonon's contribution to the spectral function is a delta peak, while anharmonic interactions broaden the frequency response. Specifically in perturbation theory, the resulting spectral density is that of a damped harmonic oscillator [4]

$$
S(\nu)=\frac{1}{\pi} \frac{\nu_{0}^{2} \Gamma}{\left(\nu^{2}-\nu_{0}^{2}\right)^{2}+\Gamma^{2} \nu^{2}} .
$$

This is also the functional form we use for fitting the peak frequencies and broadenings of the simulated MD spectra.

In neutron scattering experiments, the spectral density for a given scattering vector $\mathbf{Q}$ is directly probed. Here, the scattering amplitude in the classical setting is given by

$$
A(\mathbf{Q}, t) \propto \sum_{j} e^{-i \mathbf{Q} \mathbf{r}_{j}(t)} .
$$

In the scattering community, expanding the phase factors in the absolute-squared amplitude around the equilibrium positions is known as phonon expansion, as the $N$ th-order 
expansion term can be interpreted as being responsible for scattering events between the probe quanta and $N$ independent phonons at once [34]. Thus the linear term, corresponding to one-phonon scattering, gives just the previously introduced Eq. (1) with $\mathbf{p} \| \mathbf{Q}$, where the reduced position in reciprocal space $\mathbf{q}$ follows from the actual scattering vector $\mathbf{Q}$ by translating it into the first Brillouin zone via a reciprocal lattice vector $\mathbf{G}$. While momentum and energy conservation are responsible for the peaked shape of the one-phonon spectra, the additional degrees of freedom in $N$-phonon scattering events result in a continuously varying background. These higher-order terms are particularly relevant at large $\mathbf{Q}$-and therefore in the higher Brillouin zones probed in neutron scattering experiments-and at elevated temperatures with larger atomic displacements. Note that these issues are distinct from the peak broadening introduced by anharmonic phonon-phonon interactions-also a harmonic crystal would exhibit an appreciable background intensity in the spectral densities at high temperatures for large $\mathbf{Q}$.

In Fig. 1 we now compare the power spectra obtained from classical DFT perturbation theory (blue lines) to the one-phonon spectral functions according to DFT MD (dashed red line) obtained using Eq. (1) and the full expression [solid red line-Eq. (3)] evaluated at the scattering vector $\mathbf{Q}$ of the experiment. We see that already with respect to the one-phonon MD spectra, the damped harmonic oscillator curves according to perturbation theory typically miss low-frequency weight, and can lead to very different anharmonic broadenings (difference blue shaded). Further, specifically for phonons at large $\mathbf{Q}$ and high frequencies, the inclusion of the $N$-phonon background can result in modifications (red shaded) to the spectra of comparable significance, so that in general only the molecular dynamics spectra evaluated according to Eq. (3) give a satisfactory agreement between calculations and experiment.

While Fig. 1 was concerned with the spectral densities' shapes including contributions from all scattering channels, we now proceed to a quantitative analysis of phonon lifetimes as reflected in a broadening of the one-phonon contribution. Thus, we use Eq. (1) to compute the onephonon spectra from our DFT MD runs and subsequently fit a damped harmonic oscillator model as given by Eq. (2) to obtain frequencies $\nu_{0}$ and linewidths $\Gamma$. We do the same for the experimental spectra taking into account multiphonon background and experimental resolution as detailed in the Supplemental Material [29].

Figure 2(a) shows the $\mathbf{q}$ dependence of the phonon linewidths at $900 \mathrm{~K}$ according to perturbation theory, full $\mathrm{MD}$, and experiment. The qualitative difference between the smoothly varying MD linewidths (red symbols) and the rather jagged perturbation theory results (blue lines) is obvious. Comparing to experiment (gray symbols) settles
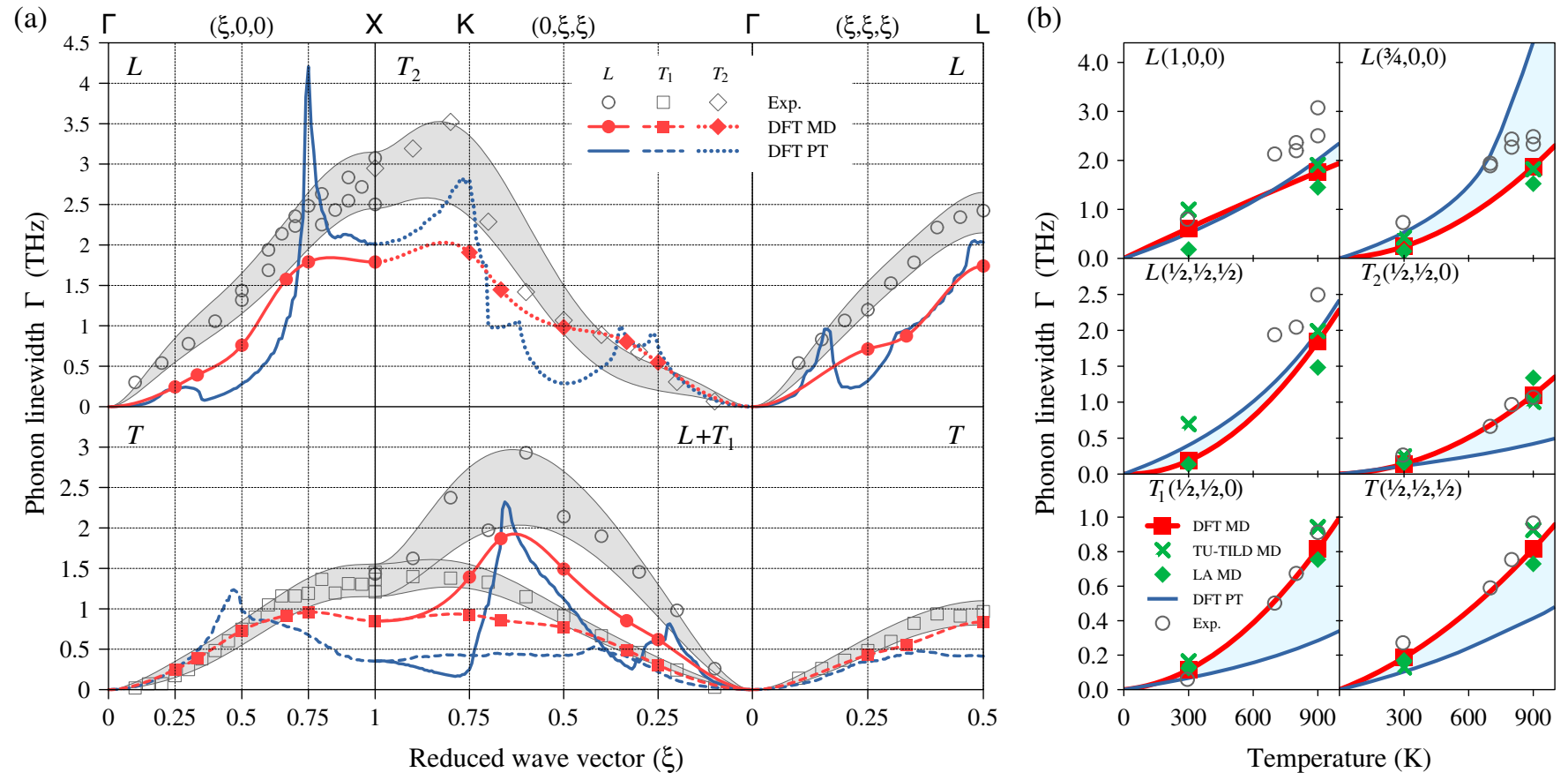

FIG. 2. Experimental phonon linewidths $\Gamma$ for $\mathrm{Al}$ (gray symbols) in comparison to perturbation theory (PT) using the third-order force constant tensors (blue lines) and results from DFT MD (GGA) in $3 \times 3 \times 3$ and $4 \times 4 \times 4$ fcc supercells [red symbols-Eq. (1)]. Here (a) shows the $\mathbf{q}$ dependence at $900 \mathrm{~K}$ for all branches and (b) the temperature dependence for selected $\mathbf{q}$ points, where in addition the linewidths according to TU-TILD [35] (green crosses) and LA [3] (green diamonds) are shown. The temperature variation in PT is due to both the (trivial) scaling with $T$ as well as a quasiharmonic interpolation of the force constants evaluated at $300 \mathrm{~K}$ and $900 \mathrm{~K}$. 
the discrepancy in favor of $\mathrm{MD}$, with a satisfactory qualitative agreement conceding an underestimation of calculated linewidths by some $30 \%$. On the other hand, the underestimation particularly of the low-frequency modes at the Brillouin zone boundary in perturbation theory is drastic. We further analyze the temperature dependence of the linewidth $\Gamma$ at selected $\mathbf{q}$ points in Fig. 2(b) which — due to the adapted scale—highlights the discrepancies between perturbation theory and MD assessments including the full anharmonicity (blue shaded). This constitutes direct evidence that the inclusion of all orders of phonon-phonon interactions is indispensable at elevated temperatures and can result in significant corrections with nontrivial behavior. Specifically, the rich $\mathbf{q}$ dependent structure of perturbation theory linewidths resulting from high-dimensional integrals over reciprocal space and reminiscent of van Hove singularities [5] seems to be completely smoothened by anharmonicity (see the Supplemental Material [29] for further arguments).

As has been noted, considering the full anharmonicity from $a b$ initio is computationally demanding for spectrally resolved quantities. Consequently, the question arises whether there are alternate approaches able to accurately predict phonon properties up to highest temperatures while keeping computational demands low. Only recently we have shown that anharmonic contributions to thermodynamic quantities such as the free energy can be obtained from DFT up to the melting temperature and to $\mathrm{meV}$ accuracy by computing merely a few DFT structures [3,35]. Here, to obtain spectrally resolved phonon quantities, we apply (i) an optimized interatomic potential fitted according to the "two-stage upsampled thermodynamic integration using Langevin dynamics" (TU-TILD) [35] method and (ii) the "local anharmonic" (LA) approximation [3]. For the former, we utilize an embedded-atom method (EAM) Hamiltonian fitted to a short DFT MD of Al containing 1500 snapshots at $900 \mathrm{~K}$ using the MEAMfit code [36]. On the other hand, the main idea of the LA approach is to map the anharmonicity of local pair interactions at $T=0 \mathrm{~K}$ by performing displacements similar to the finite displacement method but with significantly larger magnitudes to accurately reproduce the asymmetric character of the local potential, modeled as a Morse potential [3]. In the implementation used here only nearest-neighbor pairwise forces are probed by the LA approximation. We obtain the dominant longitudinal anharmonic Morse pair interaction, augmented by the transversal harmonic force constant in (001) direction, from two DFT configurations at $T=0 \mathrm{~K}$ per considered lattice constant [37]. Results on the achievable accuracy for thermodynamic quantities can be found in Refs. [3,38]. Having the optimized EAM or LA Hamiltonian at hand we perform MD and evaluate the power spectral densities as introduced above.

In Fig. 2(b) we compare the TU-TILD and LA model Hamiltonian phonon linewidths (green crosses and diamonds) to DFT (red squares). We find both approaches in very good agreement with our DFT MD results (as well as with experiment) for all probed temperatures. We note that these phenomenological models make it possible to probe much larger supercells for increasing $\mathbf{q}$ resolution (see Supplemental Material [29]).

Since the current LA implementation only considers nearest-neighbor pair interactions and has a single anharmonic degree of freedom this suggests that the major part of phonon-phonon scattering in $\mathrm{Al}$ is captured by local pairwise terms as opposed to terms involving multiple sites. To quantify this hypothesis we write the general anharmonic potential as a Taylor series in terms of the displacement $u_{\alpha}(l)$ of the atom $l$ from its equilibrium position along the Cartesian dimension $\alpha$ [5]

$$
\phi=\phi_{0}+\sum_{K \geq 2} \frac{1}{K !} \phi_{\alpha_{1}, \ldots, \alpha_{K}}\left(l_{1}, \ldots, l_{K}\right) \prod_{i=1}^{K} u_{\alpha_{i}}\left(l_{i}\right)
$$

and parametrize the potential coefficients up to quartic order $K \leq 4$ for compact clusters of atoms at sites $l_{i}$ by the forces encountered during the MD runs for DFT and LA. The resulting decomposition of the forces according to the configuration of the participating atoms $l_{i}$ is given in Fig. 3 .

Considering first the DFT results, we observe that among the quadratic interactions the nearest-neighbor term is strongest by far. This behavior carries over into the higher orders: also here the nearest-neighbor pair interactions dominate over terms involving atoms at greater separations, and surprisingly also over all multiple-site terms, e.g., the nearest-neighbor triangles $(A-B-C)$. By construction, LA obviously does not contain any interactions involving three or more atomic sites (as $A-B-C$ or $A-B-B-C$ etc.), nor beyond nearest neighbors $A-B$ (which are equivalent to $A-C$

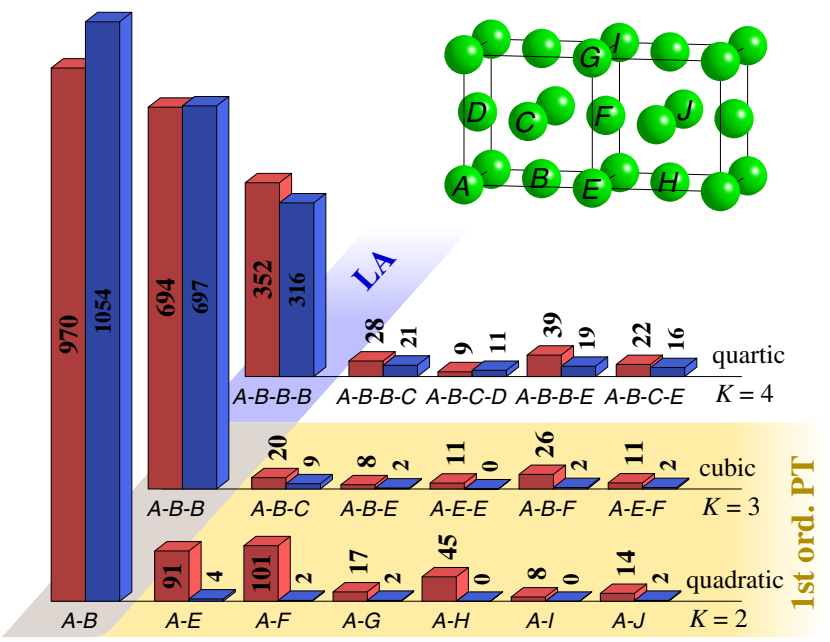

FIG. 3. Root-mean-squared forces in units of meV/Å acting on a given atom at $900 \mathrm{~K}$ for DFT (red) and LA (blue) according to Taylor expansion of the potential up to quartic order, grouped into terms according to relative coordinations of participating atoms. 
and $A-D$ ), and so the corresponding terms should be exactly zero. The reported nonzero values are therefore spurious effects due to the truncation of the fitted Taylor series. In DFT, the quadratic interactions beyond nearest neighbors, while still weak, are noticeably stronger than the spurious values in LA, and thus very likely real. Indeed, it has been known for a long time that interactions beyond first neighbors are important for describing experimental phonon dispersions satisfactorily [39]. On the other hand, higher-order interactions beyond nearest neighbors are hardly above the numerical noise as evidenced by LA.

Contrary to the prevailing opinion, we see that cubic and quartic contributions are of similar magnitude, which explains why perturbation theory-which in its most common implementations considers only interaction terms up to $K=3$-fails to explain phonon linewidths in the case of $\mathrm{Al}$ at high temperatures. Further, at high temperatures the contributions due to higher orders of the nearest-neighbor pair interactions are much larger than those due to lower orders of configurations of three atoms, or even harmonic interactions over larger separations. This implies that also in a prototypical metal such as $\mathrm{Al}$, it is the short-range repulsion due to closed shells that is primarily responsible for anharmonicity and thus derived effects such as thermal expansion and phonon heat conductivity. These findings explain the success of the LA method, which considers only pair interactions between neighboring sites, and imply that the shape of its pair potential, being given by only four parameters (three axial anharmonic and one transversal harmonic), captures the physical reality exceedingly well. In contrast, approaches that aim to evolve the potential energy surface by systematically expanding multiple-site interactions over large separation distances are not optimally suited to describe the dominant higher-order pair coefficients which govern phonon-phonon interactions.

In summary we have obtained phonon lifetimes in $\mathrm{Al}$ from experiment and $a b$ inito calculations and have compared methods of different accuracy and computational effort. We did provide direct evidence of qualitative differences in phonon linewidths between the full MD and perturbative approaches at elevated temperatures and, more importantly, have shown that the full MD is a prerequisite for computationally reproducing actual experimental spectra. Using the LA approximation we have identified the dominant mechanism that controls phonon lifetimes in $\mathrm{Al}$ at high temperatures and traced it back to the strongly anharmonic local pair interactions between neighboring atoms. Analyzing the forces in the MD runs, we have shown that multiple-site interactions-which have a central role in perturbative approaches-are an order of magnitude smaller than pair interactions even for higher orders in the perturbation expansion. Based on these insights we have demonstrated how to derive accurate potentials from DFT, which are applicable up to highest temperatures and thus can significantly contribute to our understanding of atomic-scale dynamics from experiment and theory alike.

The funding by the Deutsche Forschungsgemeinschaft SPP1713 (Grant No. HI1300/8-1) and by the European Research Council (ERC) under the European Union's Horizon 2020 research and innovation programme (Grant Agreement No. 639211) is gratefully acknowledged. A. G. acknowledges support from the SNF Flexibility Grant.

*albert.glensk@gmail.com

Present address: TU Wien, X-ray center, Getreidemarkt 9, 1060 Wien, Austria.

*michael.leitner@frm2.tum.de

[1] D. Alfè, G. D. Price, and M. J. Gillan, Phys. Rev. B 64, 045123 (2001).

[2] L. Vočadlo and D. Alfè, Phys. Rev. B 65, 214105 (2002).

[3] A. Glensk, B. Grabowski, T. Hickel, and J. Neugebauer, Phys. Rev. Lett. 114, 195901 (2015).

[4] A. A. Maradudin and A. E. Fein, Phys. Rev. 128, 2589 (1962).

[5] R. A. Cowley, Rep. Prog. Phys. 31, 123 (1968).

[6] X. Tang, C. W. Li, and B. Fultz, Phys. Rev. B 82, 184301 (2010).

[7] X. Tang and B. Fultz, Phys. Rev. B 84, 054303 (2011).

[8] F. Zhou, W. Nielson, Y. Xia, and V. Ozolinš, Phys. Rev. Lett. 113, 185501 (2014).

[9] A. Togo, L. Chaput, and I. Tanaka, Phys. Rev. B 91, 094306 (2015).

[10] T. Feng, L. Lindsay, and X. Ruan, Phys. Rev. B 96, 161201(R) (2017).

[11] J. W. L. Pang, W. J. L. Buyers, A. Chernatynskiy, M. D. Lumsden, B. C. Larson, and S. R. Phillpot, Phys. Rev. Lett. 110, 157401 (2013).

[12] P.-F. Lory et al., Nat. Commun. 8, 491 (2017).

[13] J. Menéndez and M. Cardona, Phys. Rev. B 29, 2051 (1984).

[14] A. Shukla, M. Calandra, M. d'Astuto, M. Lazzeri, F. Mauri, C. Bellin, M. Krisch, J. Karpinski, S. M. Kazakov, J. Jun, D. Daghero, and K. Parlinski, Phys. Rev. Lett. 90, 095506 (2003).

[15] K. Habicht, R. Golub, F. Mezei, B. Keimer, and T. Keller, Phys. Rev. B 69, 104301 (2004).

[16] S. Rennie, E. Lawrence Bright, J.E. Darnbrough, L. Paolasini, A. Bosak, A. D. Smith, N. Mason, G. H. Lander, and R. Springell, Phys. Rev. B 97, 224303 (2018).

[17] W. J. L. Buyers and R. A. Cowley, Phys. Rev. 180, 755 (1969).

[18] M. M. Beg and M. Nielsen, Phys. Rev. B 14, 4266 (1976).

[19] A. Larose and B. N. Brockhouse, Can. J. Phys. 54, 1990 (1976).

[20] P. Link, G. Eckold, and J. Neuhaus, Physica (Amsterdam) 276-278B, 122 (2000).

[21] O. Sobolev and J. T. Park, J. Large-Scale Res. Facil. 1, A13 (2015). 
[22] G. Kresse and J. Furthmüller, Phys. Rev. B 54, 11169 (1996).

[23] G. Kresse and J. Furthmüller, Comput. Mater. Sci. 6, 15 (1996).

[24] P. E. Blöchl, Phys. Rev. B 50, 17953 (1994).

[25] D. M. Ceperley and B. J. Alder, Phys. Rev. Lett. 45, 566 (1980).

[26] J. P. Perdew and A. Zunger, Phys. Rev. B 23, 5048 (1981).

[27] J. P. Perdew, K. Burke, and M. Ernzerhof, Phys. Rev. Lett. 77, 3865 (1996).

[28] https://atztogo.github.io/phono3py/.

[29] See Supplemental Material at http://link.aps.org/ supplemental/10.1103/PhysRevLett.123.235501 for additional information, which includes Refs. [30,31].

[30] M. J. Cooper and R. Nathans, Acta Crystallogr. 23, 357 (1967).

[31] B. Efron and R. J. Tibshirani, An Introduction to the Bootstrap, Chapman \& Hall/CRC Monographs on Statistics \& Applied Probability (CRC Press, Boca Raton, FL, 1994).
[32] Z.-Y. Ong, E. Pop, and J. Shiomi, Phys. Rev. B 84, 165418 (2011).

[33] A. J. H. McGaughey and J. M. Larkin, Annu. Rev. Heat Transfer 17, 49 (2014).

[34] G. L. Squires, Introduction to the Theory of Thermal Neutron Scattering (Cambridge University Press, Cambridge, England, 1978).

[35] A. I. Duff, T. Davey, D. Korbmacher, A. Glensk, B. Grabowski, J. Neugebauer, and M. W. Finnis, Phys. Rev. B 91, 214311 (2015).

[36] A. I. Duff, Comput. Phys. Commun. 203, 354 (2016).

[37] With an expression of $V(r)=D\left\{1-\exp \left[-a\left(r-r_{0}\right)\right]\right\}^{2}$ for the pair interactions, the LA potential is defined by $D=$ $0.258 \mathrm{eV}, a=1.437 \AA^{-1}$, and $r_{0}=2.92 \AA$, with an additional transversal linear force constant of $-0.072 \mathrm{eV} / \AA^{2}$ between nearest neighbors.

[38] A. Glensk, Ph.D. thesis, Paderborn, 2015.

[39] A. D. B. Woods, B. N. Brockhouse, R. H. March, A. T. Stewart, and R. Bowers, Phys. Rev. 128, 1112 (1962). 